\title{
Species-specific identification of Vibrio fluvialis by PCR targeted to the conserved transcriptional activation and variable membrane tether regions of the tox $R$ gene
}

Vibrio fluvialis has been reported to cause sporadic infections and outbreaks of diarrhoea in humans (Huq et al., 1980; Lesmana et al., 2002; Lai et al., 2006) and has also been isolated from marine and estuarine environments (Seidler et al., 1980; Lee et al., 1981; Lockwood et al., 1982). However, the public health significance of this pathogen has not been studied in detail due to the lack of simple and reliable diagnostic tests. Although the bacterium is known to produce several potent toxins, their role in pathogenesis is not well established (Lockwood et al., 1982; Huq et al., 1985; Kothary et al., 2003; Chakraborty et al., 2005). Information regarding virulence genes, and standard genetic markers for the identification of this organism, are not fully exploited. Despite the use of an array of biochemical tests including commercial identification systems, proper identification of $V$. fluvialis still remains a problem due to its phenotypic similarity with Aeromonas species (Seidler et al., 1980).

tox $R$ is an ancestral gene of the family Vibrionaceae which encodes a transcriptional activation domain (TAD), a transmembrane domain (TMD) and a periplasmic domain (PD) (Osorio \& Klose, 2000). Among vibrios, there is a high level of homology within the TAD of the ToxR proteins and relatively conserved homology in the TMD and PD (Osorio \& Klose, 2000). Interestingly, there is essentially no homology within the region between TAD and TMD. This region connects the TAD to the cytoplasmic membrane and it was therefore named the membrane tether region. tox $R$-based species-specific identification has been developed for Vibrio parahaemolyticus (Kim et al., 1999) and Vibrio hollisae (Vuddhakul et al., 2000). Here, we report the development of a PCR-based assay for the specific identification of $V$. fluvialis exploiting the sequence divergence within the membrane tether region of the tox $R$ gene.

Forty non-cholera Vibrio strains isolated from hospitalized patients with acute diarrhoea at the Infectious Diseases Hospital or B. C. Roy Memorial Hospital for Children, Kolkata, India, between 1998 and 2001 were included in this study. These strains were screened for Vibrio cholerae by biochemical and serological tests and by species-specific ompW PCR (Nandi et al., 2000). The oxidase-positive and ompW-negative strains were further characterized using the API $20 \mathrm{E}$ identification system (bioMérieux). Salt tolerance was determined by growing the strains at $37^{\circ} \mathrm{C}$ on nutrient agar (NA; Difco) plates containing 0 and $7 \% \mathrm{NaCl}$. For identification of the strains, $16 \mathrm{~S}$ rDNA sequencing (Microseq 500, 16S rDNA Bacterial Sequencing kit; Applied Biosystems) was performed following the manufacturer's instructions using an automated DNA sequencer (ABI Prism 310; Applied Biosystems).

To develop a PCR-based method for species-specific identification, primers targeted to the toxR gene of $V$. fluvialis were first tested with the strains that were previously identified as $V$. fluvialis by $16 \mathrm{~S}$ rDNA sequencing (Table 1). The forward (5'-GACCAGGGCTTTGAGGTGGACGAC$\left.3^{\prime}\right)$ and reverse $\left(5^{\prime}\right.$-AGGATACGGCACTTGAGTAAGACTC-3') primers were designed from the TAD and the membrane tether region of the toxR gene of $V$. fluvialis, respectively (accession no. AF170885; Osorio \& Klose, 2000). The membrane tether region is highly variable, but unique for each Vibrio species. For PCR assay, the strains were grown in Luria broth (LB; Difco) containing $1 \% \mathrm{NaCl}$ at $37^{\circ} \mathrm{C}$ with shaking at 200 r.p.m. overnight. For halophilic vibrios, the LB medium was supplemented with $3 \% \mathrm{NaCl}$. Purified genomic DNA from these strains was used as template in the PCR assay. Amplification was carried out in a thermal cycler (Applied Biosystems) with a standard PCR reaction mixture that contained $50 \mathrm{ng}$ DNA as template, $2 \cdot 5 \mu \mathrm{l} 10 \times$ PCR buffer with $15 \mathrm{mM} \mathrm{MgCl}, 0 \cdot 20 \mu \mathrm{l}(1 \mathrm{U}) \mathrm{Taq}$ Polymerase (Takara Shuzo), 2.0 $\mu 12 \cdot 5 \mathrm{mM}$ (each) deoxynucleoside triphosphate, $2 \cdot 5 \mu \mathrm{l}$ of each primer $\left(10 \mathrm{pmol} \mu \mathrm{l}^{-1}\right)$ and sterile distilled water to make the volume to $25 \mu$. The amplification conditions were initial denaturation at $94^{\circ} \mathrm{C}$ for $5 \mathrm{~min}$, followed by 30 cycles consisting of denaturation at $94^{\circ} \mathrm{C}$ for $40 \mathrm{~s}$, annealing at $65^{\circ} \mathrm{C}$ for $40 \mathrm{~s}$ and extension at $72^{\circ} \mathrm{C}$ for $1 \mathrm{~min}$. To detect the sensitivity, the VF-toxR PCR was performed with different concentrations of chromosomal DNA ranging from 10 to $60 \mathrm{ng}$. PCR products were electrophoresed through $2 \%$ agarose gel to resolve $217 \mathrm{bp}$ amplicons and visualized under UV light in a gel documentation system (Gel-Doc 2000; Bio-Rad) after ethidium bromide staining.

To confirm the specificity of the primers among members of the Vibrionaceae family, Vibrio nereis (ATCC 25917), Vibrio furnissii (ATCC 35016), Vibrio anguillarum (ATCC 19264), Vibrio vulnificus (ATCC 33816), Vibrio proteolyticus (ATCC 15338), Vibrio aestuarianus (ATCC 35048), 34 strains of $V$. cholerae including O1, O139 (10 strains each) and non-O1 non-O139 serogroups (14 strains) and 30 strains of $V$. parahaemolyticus were included in this study. As $V$. fluvialis shares biochemical properties with Aeromonas species, we included 60 clinical strains of Aeromonas, covering five species, viz. Aeromonas caviae (23 strains), Aeromonas hydrophila (18 strains), Aeromonas trota (6 strains), Aeromonas veronii (10 strains) and Aeromonas schubertii (3 strains).

All the 40 non-cholera vibrios grown in thiosulfate-citrate-bile salts-sucrose agar resembled sucrose-fermenting yellow 
Table 1. Identification results of non-cholera vibrios by different identification systems

\begin{tabular}{|c|c|c|c|}
\hline Strain & $\begin{array}{l}\text { API } 20 \mathrm{E} \text { ( } \% \text { identity; } \\
\text { profile number) }\end{array}$ & $\begin{array}{l}16 S \text { rDNA } \\
\text { sequencing }\end{array}$ & VF-toxR \\
\hline PG 39 & V. fluvialis $(81 \cdot 1 ; 3046526)$ & V. fluvialis & + \\
\hline PG 41 & $V$. fluvialis $(81 \cdot 1 ; 3046526)$ & V. fluvialis & + \\
\hline PL 45 & V. fluvialis $(83 \cdot 9 ; 3046126)$ & V. fluvialis & + \\
\hline PG 152 & V. fluvialis $(81 \cdot 1$; 3046526) & V. fluvialis & + \\
\hline PL 78/7b & Match not found (2004126) & V. fluvialis & + \\
\hline PL 169b & V. fluvialis $(83 \cdot 9 ; 3046126)$ & V. fluvialis & + \\
\hline PL 171b & V. fluvialis $(99 \cdot 8 ; 3004126)$ & V. fluvialis & + \\
\hline CRC 82 & V. fluvialis $(81 \cdot 1 ; 3046526)$ & V. fluvialis & + \\
\hline CRC 99 & V. fluvialis $(81 \cdot 1$; 3046526) & V. fluvialis & + \\
\hline CRC 100 & V. fluvialis $(99 \cdot 6 ; 3044526)$ & V. fluvialis & + \\
\hline CRC 111 & A. hydrophila $(98 \cdot 5 ; 3047527)$ & V. fluvialis & + \\
\hline CRC 159 & A. hydrophila $(97 \cdot 7 ; 3047526)$ & V. fluvialis & + \\
\hline CRC 233 & A. hydrophila $(97 \cdot 3 ; 3047126)$ & V. fluvialis & + \\
\hline AN 48 & Match not found (2046526) & $V$. fluvialis & + \\
\hline GB 987A & V. fluvialis $(83 \cdot 9 ; 3046126)$ & V. fluvialis & + \\
\hline RC 7 & $\begin{array}{l}\text { A. hydrophila }(56 \cdot 7) \text {, V. fluvialis } \\
(43 \cdot 2)(3246526)\end{array}$ & V. fluvialis & + \\
\hline RC 30 & $\begin{array}{l}\text { V. fluvialis }(73 \cdot 3) \text {, A. hydrophila } \\
(26 \cdot 6)(3046527)\end{array}$ & V. fluvialis & + \\
\hline RC 48 & V. fluvialis $(81 \cdot 1 ; 3046526)$ & $V$. fluvialis & + \\
\hline RC 50 & V. fluvialis $(99 \cdot 6 ; 3044526)$ & V. fluvialis & + \\
\hline RC 82 & V. fluvialis $(99 \cdot 6 ; 3044126)$ & V. fluvialis & + \\
\hline RC 110 & $\mathrm{ND}$ & V. fluvialis & + \\
\hline RC 152 & V. fluvialis $(99 \cdot 6 ; 3044126)$ & V. fluvialis & + \\
\hline NT 4189 & V. fluvialis $(81 \cdot 1 ; 3046526)$ & V. fluvialis & + \\
\hline VTE 624 & V. fluvialis $(83 \cdot 9 ; 3046126)$ & V. fluvialis & + \\
\hline GB 1136 & V. fluvialis $(83 \cdot 9 ; 3046126)$ & V. fluvialis & + \\
\hline CRC 2 & V. fluvialis $(81 \cdot 1 ; 3046526)$ & V. vulnificus & + \\
\hline CRC 15 & $\mathrm{ND}$ & V. vulnificus & + \\
\hline CRC 126 & V. fluvialis $(99 \cdot 6 ; 3044526)$ & V. vulnificus & + \\
\hline CRC 175 & V. fluvialis $(99 \cdot 6$; 3044126) & V. vulnificus & + \\
\hline GB 898 & V. fluvialis $(83 \cdot 9 ; 3046126)$ & V. vulnificus & + \\
\hline GB 973 & V. fluvialis $(83 \cdot 9 ; 3046126)$ & V. vulnificus & + \\
\hline RC 6 & V. fluvialis $(98 \cdot 3 ; 3244126)$ & V. vulnificus & + \\
\hline RC 96 & $\begin{array}{l}\text { V. fluvialis }(54 \cdot 9) \text {, A. hydrophila } \\
(43 \cdot 3)(2046126)\end{array}$ & V. vulnificus & + \\
\hline RC 108 & V. fluvialis $(81 \cdot 8 ; 3046526)$ & V. vulnificus & + \\
\hline RC 111 & V. fluvialis $(83 \cdot 9 ; 3046126)$ & V. vulnificus & + \\
\hline RC 112 & V. fluvialis (98.9; 3044137) & V. vulnificus & + \\
\hline RC 137 & V. fluvialis $(83 \cdot 9 ; 3046126)$ & V. vulnificus & + \\
\hline RC 203 & ND & V. vulnificus & + \\
\hline RC 218 & ND & V. vulnificus & + \\
\hline RC 237 & ND & V. vulnificus & + \\
\hline
\end{tabular}

ND, Not done.

colonies of $V$. cholerae. Further characterization showed that they were oxidase-positive, grew on NA containing $7 \% \mathrm{NaCl}$ but not in the absence of $\mathrm{NaCl}$, and were negative in the ompW PCR. The salt tolerance test is very important for differentiation of $V$. fluvialis from
Aeromonas species (Seidler et al., 1980; Lee et al., 1981) as Aeromonas species cannot grow in the presence of $7 \% \mathrm{NaCl}$. When we tested 35 non-cholera vibrios by the API $20 \mathrm{E}$ system, there were variations in the identification result (Table 1). Overall, $27(77 \cdot 1 \%)$ out of 35 tested strains were identified as $V$. fluvialis, $3(8 \cdot 6 \%)$ as A. hydrophila and for 2 strains the exact match was not found in the index of the manufacturer (Table 1). In addition, the API 20E system gave ambiguous identity (Table 1) with 3 strains (RC7, RC30 and RC96). 
Table 2. Comparison of $16 \mathrm{~S}$ rDNA sequencing results of non-cholera vibrios

\begin{tabular}{|c|c|c|c|}
\hline Strain & \multicolumn{3}{|c|}{ 16S rDNA sequencing (bit score; nucleotide match; \% homology) } \\
\hline CRC 15 & V. vulnificus $(981 ; 495 / 495 ; 100)$ & V. fluvialis $(975 ; 494 / 495 ; 99)$ & \\
\hline CRC 126 & V. vulnificus $(989 ; 499 / 499 ; 100)$ & V. fluvialis $(975 ; 497 / 499 ; 99)$ & \\
\hline CRC 175 & V. vulnificus $(1031 ; 520 / 520 ; 100)$ & V. fluvialis $(1025 ; 519 / 520 ; 99)$ & \\
\hline RC 6 & V. vulnificus $(906 ; 457 / 457 ; 100)$ & V. furnissii $(898 ; 456 / 457 ; 99)$ & V. fluvialis $(898 ; 456 / 457 ; 99)$ \\
\hline RC 96 & V. vulnificus $(1059 ; 537 / 538 ; 99)$ & V. fluvialis $(1045 ; 535 / 538 ; 99)$ & \\
\hline RC 108 & V. vulnificus $(1060 ; 535 / 536 ; 99)$ & V. fluvialis $(1046 ; 533 / 536 ; 99)$ & \\
\hline RC 111 & V. vulnificus $(918 ; 493 / 499 ; 98)$ & V. furnissii $(910 ; 492 / 499) ; 98)$ & V. fluvialis (904; 469/472; 99) \\
\hline RC 112 & V. vulnificus $(1051 ; 536 / 538 ; 99)$ & V. fluvialis $(1037 ; 534 / 538 ; 99)$ & \\
\hline RC 237 & V. vulnificus $(1051 ; 536 / 538 ; 99)$ & V. furnissii $(1043 ; 535 / 538 ; 99)$ & V. fluvialis $(1037 ; 534 / 538 ; 99)$ \\
\hline
\end{tabular}

$16 \mathrm{~S}$ rDNA sequence analysis is based on the matching of the sequence from the amplified fragment with the sequences available in the data bank, which is represented by a score system, nucleotide match and per cent homology. The $16 \mathrm{~S}$ rDNA sequencing identified $25(62 \cdot 5 \%)$ strains as $V$. fluvialis (Table 1 ) and 15 $(37 \cdot 5 \%)$ strains as $V$. vulnificus in the first identification score (Table 2). However, in the second and third identification scores, 12 and 3 strains, respectively, were identified as $V$. fluvialis (Table 2). The scores were based on the maximal match of sequenced nucleotides and a difference in 1 nucleotide altered the score value to 8 . Due to such small variation in the nucleotides, 16S rDNA sequencing and the BLAST search (http://www.ncbi.nlm.nih.gov) identified 15 strains as $V$. vulnificus in the first score with 99-100\% identity, of which 12 and 3 were $V$. fluvialis and $V$. furnissii in the second score with 97-99\% and 98-99\% identity, respectively (Table 2 ). The API profiles of 11 strains did not show any match with $V$. vulnificus or $V$. furnissii (Table 1).

The 16S rRNA nucleotide sequence comparison method is a useful molecular tool for the identification and taxonomy of different bacterial species. The minimal difference in the 16S rRNA sequences among vibrios (Kita-Tsukamoto et al., 1993; Ruimy et al., 1994) sometimes makes their identification inconclusive. Moreover, this time-consuming identification method is not cost-effective, as it needs an automated sequencer and software.

Considering the association of $V$. fluvialis with disease (Huq et al., 1980, 1985), it was important to develop a PCR-based identification method that would be useful for routine analysis. In the VF-toxR PCR, $25 \mathrm{~V}$. fluvialis strains that were identified by $16 \mathrm{~S}$ rDNA sequencing gave an expected amplicon of $217 \mathrm{bp}$ (Table 1). Sequence analysis of the $217 \mathrm{bp}$ amplicon from a representative strain confirmed the identity of $V$. fluvialis toxR, encoding the transmembrane regulatory protein (data not shown). Fifteen strains that were presumptively identified (based on API profile and/or $\mathrm{NaCl}$ tolerance test) as $V$. fluvialis were also positive in the VF-toxR PCR.

The VF-toxR PCR showed $100 \%$ sensitivity for all the $V$. fluvialis strains tested. Although 50 ng chromosomal DNA was used in the routine PCR assay, 10 ng DNA was found to give a positive result. More importantly, the VF-toxR primers did not give a positive amplicon with Aeromonas strains or with other vibrios. The observation that the VF-toxR primers can differentiate between $V$. fluvialis and Aeromonas strains is significant due to the fact that these two groups of organisms share common biochemical properties, which often leads to misidentification. This PCR method would be useful for rapid identification of $V$. fluvialis strains and may play a role in establishing the public health and clinical significance of this organism. The utility of this PCR assay should be evaluated in terms of direct detection of $V$. fluvialis from stool specimens.

\section{Acknowledgements}

This work was supported in part by the Japan International Co-operation Agency (JICA/NICED project 054-1061-E-0) and grant-in-aid from the Ministry of Health, Labor and Welfare of Japan (Project, H 17- Shinkou -3).

\section{Rupa Chakraborty, ${ }^{1}$ Sutapa Sinha, ${ }^{1}$ Asish K. Mukhopadhyay, ${ }^{1}$ Masahiro Asakura, ${ }^{2}$ Shinji Yamasaki, ${ }^{2}$ S. K. Bhattacharya, ${ }^{1}$ G. Balakrish Nair ${ }^{3}$ and T. Ramamurthy ${ }^{1}$}

${ }^{1}$ National Institute of Cholera and Enteric Diseases, P-33, CIT Road, Scheme XM, Beliaghata, Kolkata 700010, India

${ }^{2}$ Graduate School of Life and Environmental Sciences, Osaka Prefecture University, Osaka, Japan

${ }^{3}$ International Center for Diarrhoeal Disease Research Centre, Dhaka, Bangladesh

Correspondence: T. Ramamurthy (tramu@vsnl.net)

Chakraborty, R., Chakraborty, S., De, K. \& 7 other authors (2005). Cytotoxic and cell vacuolating activity of Vibrio fluvialis isolated 
from pediatric patients with diarrhoea. $J$ Med Microbiol 54, 707-716.

Huq, M. I., Alm, A. K., Brenner, D. J. \& Morris, G. K. (1980). Isolation of Vibrio-like group, EF-6, from patients with diarrhoea. J Clin Microbiol 11, 621-624.

Huq, M. I., Aziz, K. M. \& Colwell, R. R. (1985). Enterotoxigenic properties of Vibrio fluvialis (Group F Vibrio) isolated from clinical and environmental sources. J Diarrhoeal Dis Res 3, 96-99.

Kim, Y. B., Okuda, J., Matsumoto, C., Takahashi, N., Hashimoto, S. \& Nishibichi, M. (1999). Identification of Vibrio parahaemolyticus strains at the species level by PCR targeted to the toxR gene. J Clin Microbiol 37, 1173-1177.

Kita-Tsukamoto, K., Oyaizu, H., Nanba, K. \& Shimidu, U. (1993). Phylogenetic relationships of marine bacteria, mainly members of the family Vibrionaceae, determined on the basis of $16 \mathrm{~S}$ rRNA sequence. Int J Syst Bacteriol 43, 8-19.

Kothary, M. H., Lowman, H., McCardell, B. A. \& Tall, B. D. (2003). Purification and characterization of enterotoxigenic El Tor like hemolysin produced by Vibrio fluvialis. Infect Immun 71, 3213-3220.

Lai, C. H., Hwang, C. K., Chin, C., Lin, H. H., Wong, W. W. \& Liu, C. Y. (2006). Severe watery diarrhoea and bacteraemia caused by Vibrio fluvialis: a first case report. J Infect 52, 95-98.

Lee, J. V., Shread, P., Furniss, A. L. \& Bryant, T. N. (1981). Taxonomy and description of Vibrio fluvialis sp. nov. (synonym group F Vibrios, Group EF-6). J Appl Bacteriol 50, 73-94.

Lesmana, M., Subekti, D. S., Tjaniadi, P., Simanjuntak, C. H., Punjabi, N. H., Campbell, J. R. \& Oyofo, B. A. (2002). Spectrum of Vibrio species associated with acute diarrhoea in North Jakarta, Indonesia. Diagn Microbiol Infect Dis 43, 91-97.

Lockwood, D. E., Kreger, A. S. \& Richardson, S. H. (1982). Detection of toxins produced by Vibrio fluvialis. Infect Immun 35, 702-708.

Nandi, B., Nandy, R. K., Mukhopadhyay, S., Nair, G. B., Shimada, T. \& Ghose, A. C. (2000). Rapid method for species-specific identification of Vibrio cholerae using primers targeted to the gene of outer membrane protein ompW. J Clin Microbiol 38, 4145-4151.
Osorio, C. R. \& Klose, K. E. (2000). A region of the transmembrane regulatory protein ToxR that tethers the transcriptional activation domain to the cytoplasmic membrane displays wide divergence among Vibrio species. J Bacteriol 182, 526-528.

Ruimy, R., Breittmayer, V., Elbaze, P., Lafay, B., Boussemart, O., Gauthier, M. \& Christine, R. (1994). Phylogenetic analysis and assessment of the genera Vibrio, Photobacterium, Aeromonas and Plesiomonas deduced from small-subunit rRNA sequences. Int J Syst Bacteriol 44, 416-426.

Seidler, R. J., Allen, D. A., Colwell, R. R., Joseph, S. W. \& Daily, O. P. (1980). Biochemical characteristics and virulence of environmental group $\mathrm{F}$ bacteria isolated in the United States. Appl Environ Microbiol 40, 715-720.

Vuddhakul, V., Nakai, T., Matsumoto, C., Oh, T., Nishino, T., Chen, C. H., Nishibuchi, M. \& Okuda, J. (2000). Analysis of gyrB and toxR gene sequences of Vibrio hollisae and development of gyrB and toxR targeted PCR methods for isolation of $V$. hollisae from the environment and its identification. Appl Environ Microbiol 66, 3506-3514. 